\title{
Physical activity is a major contributor to the ultra low frequency components of heart rate variability
}

\author{
J M Serrador, H C Finlayson, R L Hughson
}

\begin{abstract}
Objective-To investigate the link between changes in level of physical activity and the pattern of heart rate variability during long term ambulatory monitoring.

Design-Heart rate variability was measured simultaneously with a quantitative indicator of muscle activity by electromyography (EMG) in five men and five women while they did activities typical of daily life or while they rested for 2-3 hours. Spectral and cross spectral analyses were performed on both variables with standard fast Fourier transform.

Results-There was a marked reduction in spectral power in the ultra low frequency band $(<0.003 \mathrm{~Hz})$ on going from active to rest conditions for both heart rate variability (men 6187 (1801) $v 410(89)$ $\mathrm{ms}^{2} / \mathrm{Hz}$; women 4056 (1161) v 2094 (801), mean (SEM); $p<0.01)$ and EMG $(\mathrm{p}<0.001)$. Cross spectral analysis showed a strong positive gain between the EMG and heart rate variability signal that was virtually eliminated in the resting condition $(p<0.01)$. A sex-by-condition effect $(p=0.06)$ was noted with a reduction in total spectral power for heart rate variability during rest in men, while it increased slightly in women.

Conclusions-There is a quantitative link between muscle activation and heart rate variability in the lowest frequency band. Voluntary restriction of physical activity in healthy young subjects caused marked reduction in spectral power in the lowest frequency band which is often used to assess patient prognosis. The findings strongly suggest that studies of ambulatory heart rate variability should always include an indication of physical activity patterns.

(Heart 1999;82:e9)
\end{abstract}

Keywords: spectral analysis; electromyography; Holter monitoring; sex effect

Faculty of Applied

Health Sciences,

University of Waterloo,

Waterloo, Ontario

N2L 3G1, Canada

J M Serrador

H C Finlayson

R L Hughson

Correspondence to: Dr Hughson

email: hughson@

healthy.uwaterloo.ca

Accepted for publication 16 August 1999 has been adopted in the recent task force report. ${ }^{6}$ There is considerable evidence that the power in the $\mathrm{HF}$ band is a function of variation in parasympathetic nervous system activity, and that modulation of both parasympathetic and sympathetic activity contributes to the LF band..$^{6-9}$ However, there is no consensus on the origin of heart rate variability in the VLF and ULF bands. ${ }^{6}{ }^{10}$ This should be a major concern as most of the spectral power is contained in these lowest frequency bands ${ }^{1}$ and clinical interpretations rely heavily on changes in low frequency power. ${ }^{1-5}$

Suggested causes of heart rate variability in the ULF and VLF bands include variations in hormones of the renin-angiotensin-aldosterone system, ${ }^{7}$ temperature regulation, ${ }^{11}$ circulating catecholamines, ${ }^{12}$ or changes in physical activity. ${ }^{13}{ }^{14}$ Because the latter is certain to change during a 24 hour recording that includes sleep as well as normal daily activities, it is essential that a quantitative approach be used to explore the interrelations between physical activity and heart rate variability.

Osterhues et al have shown that patients with coronary artery disease who were confined to bed had markedly reduced time domain indicators of heart rate variability in their 24 hour Holter monitoring compared with ambulatory patients. ${ }^{14}$ In contrast, there were no differences in heart rate variability between the ambulatory patient population and healthy, age matched control subjects. Bernardi et al had healthy subjects complete both rhythmic activity (alternating rest and mild exercise) and spontaneous activity for comparison with quiet rest. ${ }^{13}$ They found that heart rate variability in the VLF band was increased by the physical activity during a one hour collection period.

The purposes of the present study were to employ a quantitative method of assessing the level of physical activity and to correlate the magnitude of physical activity with heart rate variability in the ULF band. Quantitative indications of muscle activity were obtained by recording muscle electomyographic activity (EMG) from four muscles continuously during a three hour period in which healthy young subjects were asked to move through a series of prescribed tasks typical of normal daily life. These same healthy subjects were also asked to sit quietly and read for comparison with the results during physical activity. We hypothesised that cross spectral analysis would indicate a high correlation between physical activity and spectral power in the ULF and VLF bands. 


\section{Methods}

SUBJECTS

Ten healthy subjects, five men and five women, volunteered for this study. Physical characteristics of the male subjects were (mean (SEM)): age 25.2 (1.9) years, height $173.8(6.4) \mathrm{cm}$, and weight $70.2(2.7) \mathrm{kg}$; and of the female subjects, age 24.0 (0.9) years, height 171.4 $(2.8) \mathrm{cm}$, and weight $62.0(1.6) \mathrm{kg}$. After receiving a complete description of the experimental protocol and potential risks, each subject signed a consent form approved by the Office of Human Research at the University of Waterloo. Subjects were asked to avoid heavy exercise and refrain from consumption of caffeine or alcohol for the 24 hours before each session.

EXPERIMENTAL DESIGN

Subjects participated in two separate three hour laboratory sessions, an active and a resting condition administered in a random order. RR interval was obtained from a standard three lead placement with an ECG, recorded on a personal computer after $\mathrm{A} / \mathrm{D}$ conversion at $1000 \mathrm{~Hz}$. Surface electromyographic activity (EMG) was measured on the right side in the rectus femoris, erector spinae, anterior deltoid, and flexor digitorum superficialis using standard lead placement. ${ }^{15} \mathrm{~A}$ portable EMG unit (ME 3000 Professional; Mega Electronics, Kuopio, Finland) was used to collect and rectify the raw signal. The EMG data were stored as one second averages for each muscle. All EMG data were normalised with respect to the electrical activity during a maximum voluntary contraction for each muscle. Total EMG activity was obtained by summing
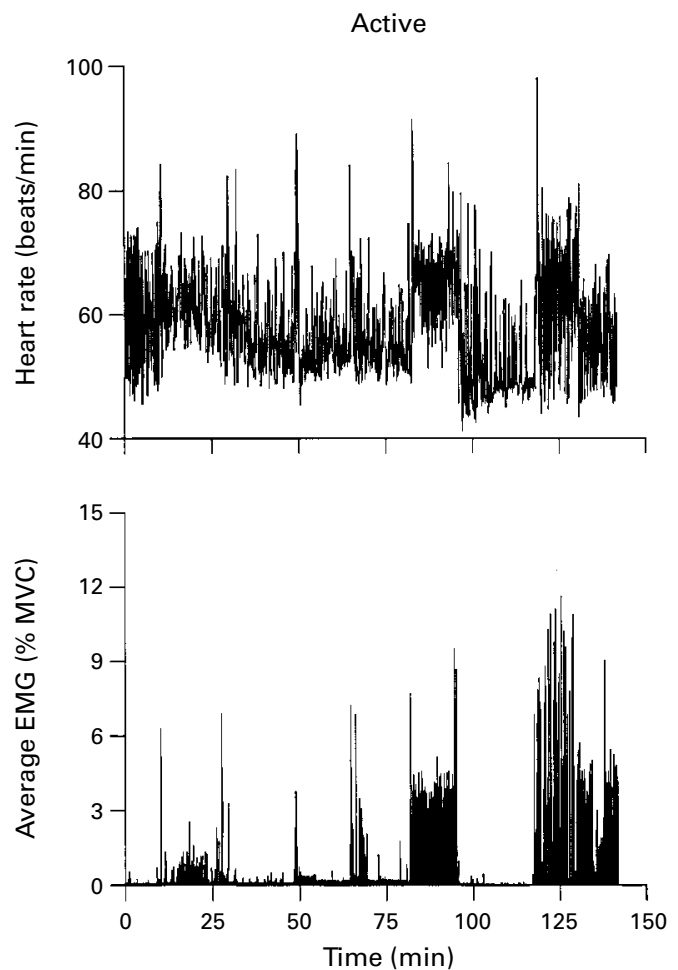

the normalised values from the four muscles according to the weighting: total $\mathrm{EMG}=0.3$ rectus femoris +0.5 erector spinae +0.1 anterior deltoid +0.1 flexor digitorum superficialis.

The active condition consisted of a series of light activities. Each subject completed sorting through a group of magazines for specific articles (15 minutes), reading quietly in a seated position (15 minutes), typing on keyboard (15 minutes), hand writing (15 minutes), walking (15 minutes), resting with legs elevated (20 minutes), picking up objects from floor (10 minutes), completing jigsaw puzzle (15 minutes), and playing a computer game (Tetris) on a personal computer (20 minutes).

The resting condition consisted of subjects seated in a quiet room reading for up to 180 minutes to obtain the same number of heart beats as in the active trial. Subjects were requested to avoid major movements but were permitted to turn pages, reach, and shift positions within the chair. This allowed for greatly decreased amplitude of input from physical activity to cardiovascular control.

Fast Fourier transforms using CooleyTukey's FFT algorithm were performed on both the RR intervals and weighted total EMG data. The EMG signal, originally sampled at 1 $\mathrm{Hz}$, was resampled at the time of each heart beat by a zero order hold. Cross spectral gain between RR interval and average EMG was calculated with the same algorithm.

\section{STATISTICAL ANALYSIS}

Data are expressed as mean (SEM). A repeated measures analysis of variance was used to examine the main effects of condition and sex and their interaction for each frequency band
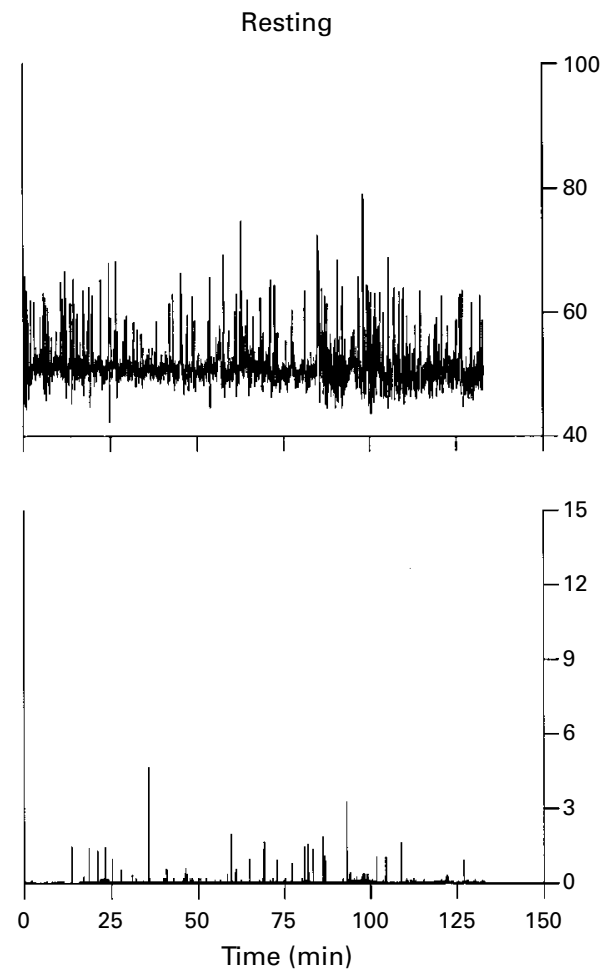

Figure 1 Beat by beat changes in heart rate and corresponding changes in EMG activity for a typical female subject during both her active and resting trials. EMG data are the weighted average from four muscles, as outlined in Methods. 
on the dependent variables RR interval, EMG activity, and cross spectral gain. Significant interactions were examined for condition effects within the sexes. Results were considered significant at $\mathrm{p}<0.05$.

\section{Results}

The mean heart rate observed across the active test day was significantly greater than that on the rest day (66.0 (3.3) v 59.0 (2.9) beats/min, $\mathrm{p}<0.02)$. Likewise, the mean EMG activity was greater on the active than the rest day $(2.73$ $(0.79) \quad 00.55$ (0.15) \% MVC, $\mathrm{p}<0.05)$. Figure 1 shows beat by beat heart rate and EMG for a typical subject during active and resting trials.

\section{EFFECTS OF ACTIVITY}

There was a decrease in total power for heart rate variability from the active to resting condition (9924 (1775) v $7164(1765) \mathrm{ms}^{2} / \mathrm{Hz}$, $p=0.30$ ), although these changes were not significant. All subjects had reductions in the total
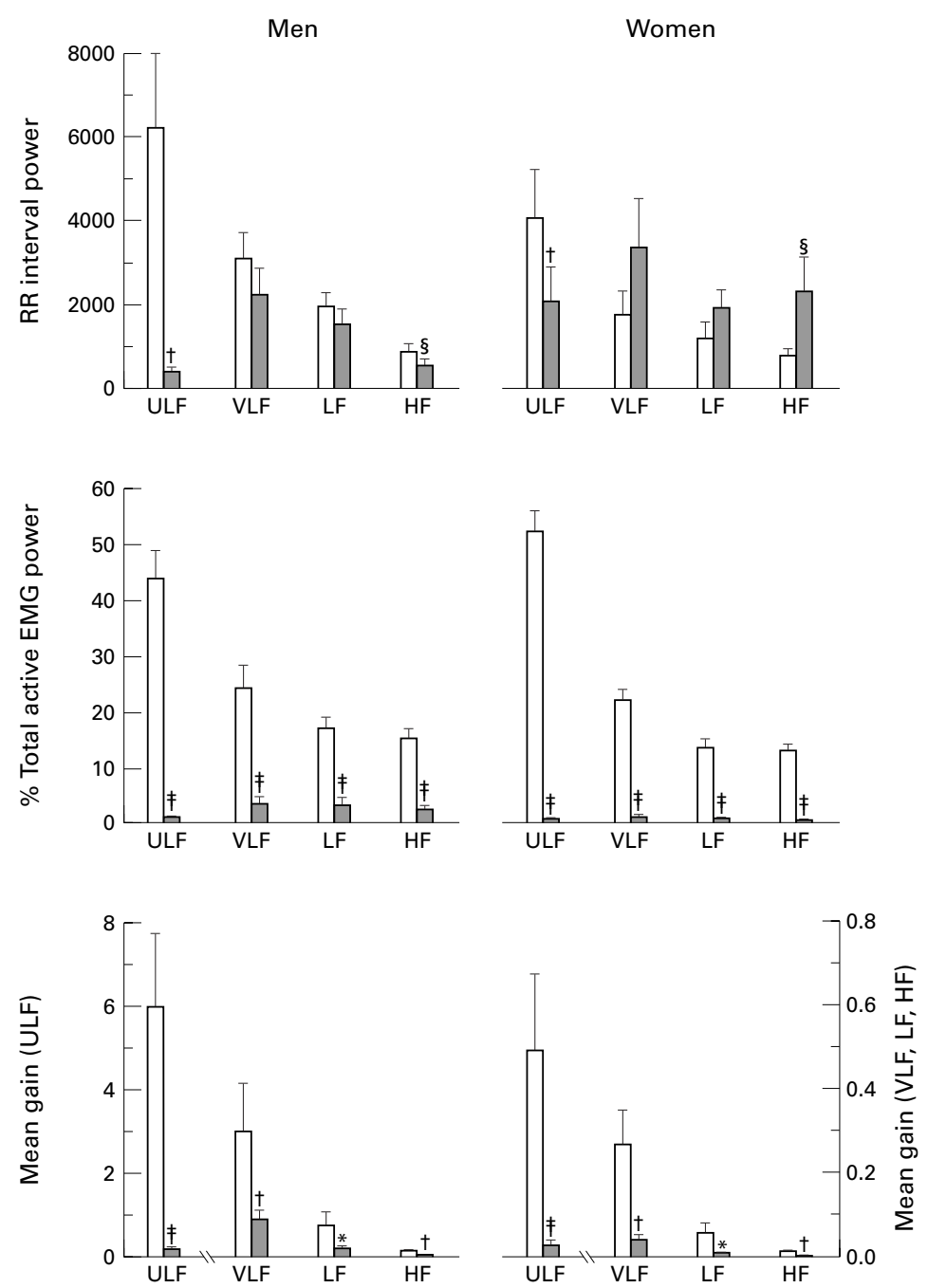

Figure 2 Changes in spectral power for RR interval $\left(\mathrm{ms}^{2} / \mathrm{Hz}\right)$ and $\mathrm{EMG}(\% \mathrm{MVC} / \mathrm{Hz})$ in specific frequency bands from active and resting conditions for men and women. Total power spectral density for the RR interval (upper panels). Total spectral power of EMG activity normalised to total spectral power for the active trial (middle panels). Mean gain for those frequencies at which coherence was greater than 0.5 (lower panels, note the change in scale for $V L F, L F$, and $H F$ frequency bands). All values are means, error bars $=S E M$. Significant difference between active and resting: ${ }^{\star} p<0.05, \dagger p<0.01, \ddagger p<0.005$.

Significant sex interaction, $\$ p<0.05$. power for the EMG (expressed relative to the percentage of maximum voluntary contraction, $\% \mathrm{MVC}^{2} / \mathrm{Hz}$ ) from the active to resting condition (23.9 (13.2) $v 0.30$ (0.08), $\mathrm{p}<0.001)$.

There was a dramatic decrease in ULF power for RR interval on going from active to resting conditions in both male and female subjects (fig $2, \mathrm{p}<0.01$ ). Significant decreases were also found in both per cent total active EMG power $(p<0.001)$ and mean gain of the cross spectral relation for EMG on RR interval in the ULF band ( $p<0.01$, fig 2).

In the VLF, LF, and HF bands, there were no significant changes in spectral power for RR interval in active versus resting conditions. In both male and female subjects the resting conditions caused significant decreases in the per cent total active EMG power in all three bands $(\mathrm{p}<0.005)$ and in mean gain (VLF, $\mathrm{p}<0.01$; LF, $\mathrm{p}<0.05 ; \mathrm{HF}, \mathrm{p}<0.01$ ). It should be noted that, although there were significant reductions in mean gain across all frequency bands, the ULF values were at least 10 times the values in any other band.

SEX EFFECTS

There was no significant sex effect for RR interval total power when data within each sex were pooled for active and resting conditions. However, when the data were analysed separately for sex and for activity, the interaction effect approached significance $(p=0.06)$. That is, the men had a marked reduction in RR interval total power on going from active to resting conditions (12068 (2703) $v 4577$ (1247) $\mathrm{ms}^{2} / \mathrm{Hz}$ ) compared with the women, who had a slight increase (7779 (2138) v 9671 (3052) $\mathrm{ms}^{2} / \mathrm{Hz}$ ).

The sex-by-activity interaction in the $\mathrm{HF}$ band of the RR interval power was significant ( $p<0.05$, fig 2). Men decreased HF power from active to resting tests, while women increased power in this frequency band.

\section{Discussion}

Considerable emphasis has been placed on the reduction in heart rate variability seen in the ULF and VLF bands of patients after acute myocardial infarction because of its link to patient prognosis. ${ }^{1-5}$ As indicated by the recent task force report, the origin of ULF and VLF components of heart rate variability modulation is unknown. ${ }^{6}$ Our current results, showing a strong correlation between quantity of muscle activity and magnitude of ULF spectral power of heart rate variability, add to our understanding of this component of heart rate variability. The results go beyond those from recent studies that provided qualitative data on a link between physical activity and heart rate variability. ${ }^{13}{ }^{14}$ Modulation of the level of energy expenditure that occurs as a normal part of daily life is almost certainly a major contributor to power in the ULF band of the long term record of heart rate variability.

\section{QUANTIFICATION OF MUSCLE ACTIVITY AND} HEART RATE VARIABILITY

We monitored the recruitment of skeletal muscles by surface EMG recording from four mus- 
cles during the physically active and resting trials. It has been well documented that EMG provides a valid index of the extent of muscle fibre activation. ${ }^{16}$ Indeed, in studies of daily energy expenditure, heart rate is often used as an indicator of muscle activation because of the linear relation between heart rate and work rate as tested on a cycle ergometer or treadmill. ${ }^{17}$ However, our goal was to examine the relation between normal daily activities, in which work rate cannot be specifically defined, and heart rate. There is no published guidance on how to sum the activity of different muscle groups as input to the cardiovascular control centre. For dynamic exercise, total mass of active muscle appears to be the key factor. ${ }^{18}$ For static contractions, the percentage of MVC is also important in determining both the heart rate and blood pressure responses. ${ }^{18}$ We have used a summation of the muscle activity, in which the leg muscle (rectus femoris) and muscles in the lower back (erector spinae) were taken to represent activation of large muscle mass, while the upper arm (deltoid), and forearm (flexor digitorum superficialis) were considerably smaller muscles. This scheme appeared to provide a useful index of total muscle activity as seen in fig 1.

The physical activity tasks used in this study were selected to be typical of light daily activities performed by most individuals during a normal day. There were no periods of strenuous exercise, as indicated by the relatively low heart rate and EMG activity for a single subject (fig 1). The total test duration of 2.25-3 hours was selected because it allowed for adequate sampling to test heart rate variability into the ULF band. Given the guideline that the recording should last at least 10 times the wavelength of the lower frequency bound, ${ }^{19}$ a period of 2.25 hours is equivalent to $8100 \mathrm{sec}-$ onds, so that this is 10 times a maximum period of $810 \mathrm{~s}$, or $0.001 \mathrm{~Hz}$. This is less than the cut off of $0.003 \mathrm{~Hz}$ for the ULF band. While it might be argued that the imposition of a specific order of physical tasks could induce the pattern of heart rate variability, it is logical that our normal daily activities go from periods of little or no activity to periods of moderate activity in random order. As long as there are variations in physical activity, the order will not have a major impact on the outcome of spectral analysis. A factor that will influence the distribution of the spectral power is the duration of each activity. Just as Bernardi et al had all power compressed into the VLF band because of their repetitions of exercise at three minute intervals, ${ }^{13}$ power in our current study would be expected to be distributed within the ULF and VLF bands because of the 15-20 minute duration of each activity segment.

A critical issue in the application of spectral analysis with fast Fourier transform is that of stationariness. ${ }^{6}$ In the analysis of short term heart rate variability, it is critical that stationarity be obtained, as the power will dominate in the lower frequency bands and greatly influence the interpretation of indicators of parasympathetic and sympathetic tone. For long term recordings of 24 hour heart rate variability, it is impossible to achieve stationarity. In spite of this, 24 hour recording with spectral analysis has been advocated in two recent reports on standardisation of methodology. ${ }^{6}{ }^{10}$ Stationarity is not present across the full period of data collection in the current study. However, to allow us to make comparisons with existing data and to show the limitations of the recommendations of these recent reports, we have continued with spectral analysis.

DETERMINANTS OF HEART RATE VARIABILITY

The origin of the majority of heart rate variability in the HF and LF bands is well understood to be a function of modulation of parasympathetic and sympathetic nervous system activity associated with breathing movements and changes in arterial blood pressure. ${ }^{6-102021}$ The origin of heart rate variability in the ULF and VLF bands has been the subject of only limited study. One suggested cause has been variation in the level of hormones of the renin-angiotensin system, ${ }^{7}$ although heart rate variability was not modified when this hormone system was altered by head down tilt. ${ }^{22}$ Other potential regulators include variations in thermoregulation, ${ }^{11}$ plasma catecholamines, ${ }^{12}$ peripheral chemoreceptors, ${ }^{23}$ and physical activity. ${ }^{13}{ }^{14}$

Bernardi et al were the first to show a link between activity patterns and heart rate variability. ${ }^{13}$ The three minute pattern of activity forced an oscillation at $0.0056 \mathrm{~Hz}$ in the VLF band. Their results indicated that VLF power during rhythmic or spontaneous activity increased three- to fivefold over VLF power during quiet rest. In the current study where subjects were monitored for three hours, physical activity had a predominant effect on the ULF band. Patients with coronary artery disease who were allowed to perform their normal daily activities had significantly increased standard deviation of all normal to normal heart beat intervals (SDNN) in comparison with matched patients who were confined to bed for the 24 hour period. ${ }^{14}$ In contrast, there was no difference in SDNN for the active patients with respect to healthy age and sex matched controls who also took part in daily activities during recording.

There was a great reduction in gain calculated between EMG and heart rate variability spectral powers during the resting condition. That is, the transfer of information from activity to heart rate variability was reduced, but there was still spectral power in the ULF band of heart rate variability even in the absence of physical activity. This heart rate variability can probably be attributed to those mechanisms considered above, including blood pressure or temperature regulation and circulating hormones that would directly or indirectly modulate the firing frequency of the sinoatrial node. For all other frequency bands (VLF, LF, HF), the low values of gain indicated little transfer from physical activity to heart rate variability, at least for the activities studied. The current data show that physical activity is clearly the most important contributor to long term (ULF) human heart rate variability. 
SEX DIFFERENCES IN POWER SPECTRA

The pattern of distribution of heart rate variability spectral power was different in men and women when comparing resting and active tests. The significant sex-by-condition interaction effect for the HF power of heart rate variability was a consequence of an increase in $\mathrm{HF}$ power for women in the resting tests compared with their active tests, while the men had a slight decrease. The sex-by-condition interaction for heart rate variability total spectral power had a $p$ value of 0.06 and thus just missed the normally accepted value for significance. However, it is clear that men had a marked decrease in total power in the resting compared to the active condition, while the women had a slight increase. The major contributor to the marked reduction in total power in the men was the reduction in ULF power.

Our current results are consistent with other reports of greater resting spectral power in female compared to male subjects. ${ }^{24-26}$ In contrast, other researchers have failed to find a difference, ${ }^{2}$ or have even observed greater power in male than in female subjects in specific frequency bands from two hour recordings. ${ }^{27}$ The reason for these different findings is not immediately apparent. Yeragani et al noted no difference in spectral power between the sexes across an age range of six to 61 years ${ }^{28}$ while Jensen-Urstad et al found effects of both age and sex..$^{29}$ It is possible that fitness contributes to the differences, although greater spectral power was observed at rest in both physically trained and untrained female subjects than in their male counterparts. ${ }^{26}$ Overall, it appears that women have a greater $\mathrm{HF}$ power at rest than men and that this is probably a function of greater modulation of parasympathetic activity.

IMPLICATIONS FOR MORTALITY PREDICTION Extensive research has proposed a link between decreased heart rate variability and increased probability of cardiac mortality. Taken together with recent observations ${ }^{13}{ }^{14}$ that physical activity modifies total spectral power of heart rate variability and its distribution, caution should be exercised in future research of long term heart rate variability in patient populations. Investigations that have examined the changes in heart rate variability spectral power as a function of time after myocardial infarction ${ }^{30} 31$ should also include quantitative evaluation of the changes in physical activity levels with convalescence.

Cut off criteria have been proposed ${ }^{1}$ for classifying individuals at high risk of increased cardiac mortality according to the absolute level of heart rate variability in the ULF, VLF, and LF bands, with the spectral power in the ULF band being the best predictor. In the current study, the cardiovascular health of the subjects did not change between the active and the resting measurements, but the ULF spectral power was greatly reduced without physical activities. This result questions the strict interpretation of ULF power as a predictor of cardiac mortality.
CONCLUSIONS

In this study we provided quantitative evidence of a link between variations in physical activity and the magnitude of the variations in heart rate during long term monitoring. Short term recordings (typically 5-10 minutes in duration) should not be influenced by this factor if the subjects are kept completely motionless during data collection. ${ }^{6}$ However, other conditions under which physical activity cannot be controlled, including monitoring of fetal heart rate ${ }^{32}$ or recovery of long term heart rate variability after hip arthroplasty ${ }^{33}$ and Holter monitoring of patients with heart disease, should be viewed with caution. Further research is required to determine the effects of changes in physical activity on 24 hour ambulatory monitoring of human heart rate variability.

This research was supported by the Heart and Stroke Foundation of Ontario (T-3081). We are grateful to Mr Philip Dopp, who was supported by a Heart and Stroke Foundation Martin L Willis High School Student Scholarship, for assistance with data collection.

1 Bigger JT, Fleiss JL, Steinman RC, et al. Frequency domain measures of heart period variability and mortality after myocardial infarction. Circulation 1992;85:164-71.

2 Bigger JT, Fleiss JL, Steinman RC, et al. RR variability in healthy, middle-aged persons compared with patients with chronic coronary heart disease or recent acute myocardial infarction. Circulation 1995;91:1936-43.

3 Huikuri HV, Niemelä MJ, Ojala S, et al. Circadian rhythms of frequency domain measures of heart rate variability in of frequency domain measures of heart rate variability in healthy subjects and patients with coronary artery disease: effects of ar $121-6$.

121-6.
4 Fei L, Copie X, Malik M, et al. Short- and long-term assessment of heart rate variability for risk stratification after acute myocardial infarction. Am F Cardiol 1996;77:681-4.

5 Tsuji H, Venditti FJ, Manders ES, et al. Reduced heart rate variability and mortality risk in an elderly cohort: the Framingham Heart Study. Circulation 1994;90:878-83.

6 Task Force of the European Society of Cardiology and the North American Society of Pacing and Electrophysiology: Heart rate variability. Standards of measurement, physiological interpretation, and clinical use. Circulation 1996; 93:1043-65.

7 Akselrod S, Gordon D, Ubel FA, et al. Power spectrum analyis of heart rate fluctuation: a quantitative probe of beat-to-beat cardiovascular control. Science 1981;213:220-3.

8 Yamamoto Y, Hughson RL. On the fractal nature of heart rate variability in humans: effects of data length and $\beta$-adrenergic blockade. Am $\mathcal{F}$ Physiol Regul Integr Comp Physiol 1994;266:R40-9.

9 Yamamoto Y, Nakamura Y, Sato $\mathrm{H}$, et al. On the fractal nature of heart rate variability in humans: effects of vagal blockade. Am f Physiol Regul Integr Comp Physiol 1995;269: R830-7.

10 Berntson GG, Bigger JT, Eckberg DL, et al. Heart rate variability: Origins, methods, and interpretive caveats. Psyvariability: Origins, methods, a
chophysiology 1997;34:623-48.

11 Fleisher LA, Frank SM, Sessler DI, et al. Thermoregulation and heart rate variability. Clin Sci 1996;90:97-103.

12 Dela F, Mikines KJ, Linstow MV, et al. Heart rate and plasma catecholamines during $24 \mathrm{~h}$ of everyday life in trained and untrained men. $\mathcal{F}$ Appl Physiol 1992;73:238995.

13 Bernardi L, Valle F, Coco M, et al. Physical activity influences heart rate variability and very-low-frequency components in Holter electrocardiograms. Cardiovasc Res 1996;32:234-7.

14 Osterhues HH, Hanzel SR, Kochs M, et al. Influence of physical activity on 24-hour measurements of heart rate variability in patients with coronary artery disease. $A m \mathcal{F}$ variability in patients with
Cardiol $1997 ; 80: 1434-7$.

15 Zipp P. Recommendations for the standardization of lead positions in surface electromyography. Eur $\mathcal{F}$ Appl Physiol 1982;24:82-96.

16 Patla AE. Measurement of the electromyogram. 7 Motor Behav 1985;17:443-61

17 Moon JK, Butte NF. Combined heart rate and activity improve estimates of oxygen consumption and carbon dioxide production rates. F Appl Physiol 1996;81:1754-61.

18 Rowell LB: Human cardiovascular control. New York: Oxford University Press, 1993:396-479.

19 Vissing SF, Scherrer U, Victor RG. Relation between sympathetic outflow and vascular resistance in the calf during perturbations in central venous pressure: evidence for ing perturbations in central venous pressure: evidence for
cardiopulmonary afferent regulation of calf vascular resistcardiopulmonary afferent regulation of calf vas
ance in humans. Circ Res 1989;65:1710-17. 
20 Cooke WH, Cox JF, Diedrich AM, et al. Controlled breathing protocols probe human autonomic cardiovascular rhythms. Am f Physiol (Heart Circ Physiol) 1998;274:H709 18.

21 Taylor JA, Eckberg DL. Fundamental relations between short-term RR interval and arterial pressure oscillations in humans. Circulatio

22 Hughson RL, Maillet A, Gauquelin G, et al. Investigation of hormonal effects during 10 hour head down tilt on heart rate and blood pressure variability. $\mathcal{F}$ Appl Physiol 1995;78: 583-96.

23 Ponikowski P, Chua TP, Piepoli M, et al. Detection and significance of a discrete very low frequency rhythm in RR interval variability in chronic congestive heart failure. $A m \mathcal{F}$ Cardiol 1996;77:1320-6.

24 Ryan SM, Goldberger AL, Pincus SM, et al. Gender- and age-related differences in heart rate dynamics: are women more complex than men. f Am Coll Cardiol 1994;24:17007 .

25 Huikuri HV, Pikkujamsa SM, Airaksinen KEJ, et al. Sex-related differences in autonomic modulation of heart rate in middle-aged subjects. Circulation 1996;94:122-5.

26 Gregoire J, Tuck S, Yamamoto Y, et al. Heart rate variability at rest and exercise: influence of age, gender, and physical training. Can f Appl Physiol 1996;21:455-70.

27 Tsuji H, Larson MG, Venditti FJ, et al. Impact of reduced heart rate variability on risk for cardiac events-the Framingham Heart Study. Circulation 1996;94:2850-5.

28 Yeragani VK, Sobolewski E, Kay J, et al. Effect of age on longterm heart rate variability. Cardiovasc Res 1997;35:35-42.

29 Jensen-Urstad K, Storck N, Bouvier F, et al. Heart rate variability in healthy subjects is related to age and gender. Acta Physiol Scand 1997;160:235-41.

30 Valkama JO, Huikuri HV, Airaksinen KEJ, et al. Determinants of frequency domain measures of heart rate variability in the acute and convalescent phases of myocardial infarction. Cardiovasc Res 1994;28:1273-6.

31 Kleiger RE, Bigger JT, Bosner MS, et al. Stability over time of variables measuring heart rate variability in normal subjects. Am F Cardiol 1991;68:626-30.

32 Karin J, Hirsch M, Akselrod S. An estimate of fetal autonomic state by spectral analysis of fetal heart rate flucautonomic state by spectral analysis
tuations. Pediatr Res 1993;34:134-8.

33 Marsch SC, Skarvan K, Schaefer HG, et al. Prolonged decrease in heart rate variability after elective hip decrease in heart rate variability aft
arthroplasty. Br $\mathcal{F}$ Anaesth 1994;72:643-9. 